DOI: http://dx.doi.org/10.31048/1852.4826.v12.n1.22020

\title{
10 Correr y competir. Rituales de interacción y estilo de vida en el running
}

To run and to compete. Interaction Rituals and lifestyle in running

Gastón Julián Gil

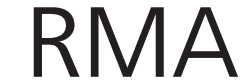

Antropología Social
CONICET, Facultad de Ciencias de la Salud y Trabajo Social, Universidad Nacional de Mar del Plata. E-mail: gasgil@hotmail.com

\section{Resumen}

En este artículo se propone un abordaje del running en la Argentina a partir del análisis de las carreras urbanas organizadas en las principales ciudades del país. Concebidas como rituales de interacción fundamentales en este estilo de vida, las carreras permiten mostrar no sólo las diferencias del running con el atletismo federado sino también algunas de las claves que explican la creciente relevancia a escala global de la opción "virtuosa" por correr. El modo en que están organizadas las carreras, el uso de las redes sociales y la masividad de los participantes permite advertir además la amplia capacidad de interpelación de públicos muy variados que ofrece este estilo de vida. Ello implica que el running proporciona un amplio menú de opciones que los actores pueden adaptar a sus expectativas y posibilidades. Estas carreras conllevan entonces verdaderos desafíos urbanísticos y de políticas de identidad para las ciudades que organizan y autorizan la realización de esta clase de eventos masivos.

Palabras clave: identidad; ritual; etnografía urbana; moralidades; deporte.

\begin{abstract}
In this article, an approach to running in Argentina is proposed from the analysis of urban races organized in the main cities of the country. Conceived as fundamental interaction rituals in this lifestyle, the races show not only the differences of running with federated athletics, but also some keys that explain the growing relevance on a global scale of the "virtuous" option for running. The way in which races are organized, the use of social networks and the massiveness of the participants also warns of the wide capacity of interpellation of very varied audiences offered by this lifestyle. This implies that running provides an extensive menu of options that the actors can adapt to their expectations and possibilities. These races then entail real urban planning challenges and identity policies for the cities that organize and authorize the realization of this kind of massive events.
\end{abstract}

Keywords: identity; ritual; urban ethnography; moralities; sport.

\section{Del salir a correr al running}

"El atletismo es deporte de negro" sentenció un entrenador de larga trayectoria en la ciudad de Mar del Plata. ${ }^{1}$ Promediaba la década de 1980 y un grupo de atletas infantiles y juveniles de un conocido club de esa ciudad escuchaba atentamente el esbozo de explicación sociológica. Como apasionado de ese deporte, el entrenador describía cómo, cada vez que detectaba alguna niña o adolescente de clase media, media alta o alta con marcadas aptitudes atléticas, su familia no dudaba en pensar en el hockey sobre césped. Del mismo modo, detallaba que para los varones tampoco la opción del atletismo parecía atractiva para el entorno familiar y siempre

\footnotetext{
1. Mar del Plata, además de ser el principal destino turístico de verano en la Argentina, mantiene una población estable de más de 600 mil habitantes, según datos del censo de 2010.
}

se pensaba en otros deportes. Más allá de representatividad de las vivencias de este profesional de la educación física y la validez de esta perspectiva de clase, el atletismo competitivo no era en aquella época, como tampoco lo es hoy, un deporte practicado mayormente por sectores sociales favorecidos en la estructura socioeconómica de la Argentina. Las competencias de atletismo de pista tampoco gozan en la actualidad de una adhesión masiva por parte de la población y no logran atraer apoyos económicos significativos por parte del Estado, las empresas o los medios de comunicación.

En contraste con el atletismo, la práctica del running ha cobrado una masividad y notoriedad vertiginosa en los últimos años, lo que puede advertirse a partir de una amplia variedad de indicadores. Los datos que arrojan las estadísticas de venta de indumentaria deportiva de 
las principales trasnacionales deportivas, los paisajes urbanos atestados de corredores en costaneras, plazas y paseos, la masividad de carreras, o la cobertura que estas competencias atléticas reciben en los medios de comunicación, son ilustrativos, además de eventuales y fructíferas fuentes de extrañamiento como para construir una investigación sobre nuevas subjetividades y estilos de vida en nuestra contemporaneidad. Puntualmente en este artículo, se realiza un abordaje comprensivo del running a partir de uno de sus rituales de interacción (Collins 2005) definitorios, las carreras. Esa dimensión ritual permite pensar a estas competencias, que pueden reunir varias decenas de miles de corredores (tanto atletas profesionales como corredores aficionados), como las principales fuentes de energía emocional que sostiene ese estilo de vida (Gil 2018). Por ende, se describirán las características y los modos en que están organizadas estas competiciones, en este caso las "de calle", en oposición a otro género de carreras conocido como trail running (Gil 2015). ${ }^{2}$ Entonces, las características de las carreras permitirán mostrar los rasgos distintivos del running en contraste con el atletismo de alta competencia, pero también sus áreas de contigüidad y continua retroalimentación. Pero además, la importancia de las carreras radica en que constituyen un marco fundamental para la definición de la identidad de quienes experimentan la apasionada opción por correr como un estilo de vida virtuoso.

Esa descripción de las carreras, su masividad y ciertos detalles de organización permitirá apreciar su centralidad en el running, como fuente prioritaria de energía emocional que garantiza no sólo la intensidad con que sus aficionados se involucran en la opción por correr sino también de su "robustez" (Latour 2008) y perspectivas de crecimiento. Colocar el foco en las carreras como rituales de interacción posibilita también visualizar la relevancia que los medios de comunicación y, sobre todo, las redes sociales tienen en la construcción del running y su masividad. Por ello es que en este artículo las carreras constituyen una de las puertas de entrada para comprender un estilo de vida que se sostiene en un conjunto denso de preceptos éticos y estéticos que trascienden la decisión puntual (incluso con cierta regularidad) de "salir a correr".

Este texto es el producto de una etnografía multi-situada (Marcus 1995) que abarca a una comunidad nacional (y en ocasiones trasnacional) de corredores cada vez más masiva que circula y se encuentra en los puntos más recónditos de la Argentina. Como parte de las labores etnográficas de mayor intensidad, se comparten entrenamientos y demás espacios de sociabilidad con integrantes de dos running teams de la ciudad de Mar del Plata. Pero el foco de indagación se expande a otros espacios geográficos, a

2 . El circuito de carreras de trail running se deja de lado de los alcances de este artículo, ya que se trata de un género de competencias con características distintivas. El trail running, también denominado vulgarmente como "de aventura" está sostenido por un denso calendario de carreras que se organizan mayormente en el interior país, en el marco de paisajes naturales en ocasiones imponentes. partir de las carreras que se celebran en distintos lugares del país y de las relaciones establecidas en esas oportunidades con corredores de diferentes procedencias, con quienes luego se mantienen contactos virtuales constantes hasta que se producen los reencuentros en otras competencias. Además, se lleva a cabo una etnografía virtual que resulta imprescindible frente a la centralidad que tienen las redes sociales en la difusión de las actividades de los corredores y sus grupos de entrenamiento, la organización de las carreras y, sobre todo, como un canal fluido para la expresión de las emociones en el acto "virtuoso" de correr.

\section{Estilo de vida y rituales de interacción}

La explosión del running forma parte de la proliferación de nuevas formas de sociabilidad en la Argentina contemporánea que puede registrarse en actividades diferenciadas, que se vinculan con prácticas deportivas, hábitos alimenticios, movimientos y manifestaciones artísticas, adopción de filosofías "exóticas", experiencias de consumo, uso de nuevas tecnologías, etc. Se trata en general de fenómenos sumamente tematizados en los medios masivos de comunicación, pero sobre todo de un alto impacto en la vida cotidiana de muchas personas. La irrupción de las nuevas tecnologías de la comunicación ha contribuido a una mayor visibilidad de estos estilos de vida por parte de comunidades que construyen, a partir de una práctica concreta y más o menos novedosa, referencias identitarias de relevancia. Ello conlleva la adopción de toda una cosmovisión que suele implicar una serie de cambios en los modos de vivir de esos actores, pero que no necesariamente generan un corte abrupto con las prácticas y representaciones que los caracterizaban con anterioridad. En el marco de esta investigación se analiza al running como parte de las transformaciones sociales que se vinculan con la concepción del cuerpo, del bienestar y del tiempo libre. El "salir a correr" que caracterizaba a solitarios deportistas, en ocasiones como complemento aeróbico de otros deportes, ha dado lugar a una práctica colectiva de notable masividad, con la difusión a gran escala de los grupos de entrenamiento o running teams. Se trata de grupos que proporcionan un marco propicio para la práctica de este deporte.

El running puede ser definido como un nuevo estilo de vida, entendido como un "conjunto de prácticas más o menos integrado que un individuo adopta no sólo porque satisfacen sus necesidades utilitarias, sino también porque dan forma material a una crónica concreta de la identidad del yo" (Giddens 1995: 106). Todo ello en el marco de una sociedad contemporánea que posibilita cada vez más recursos potenciales para establecer distinciones asociadas a los modos experimentar, representar y narrar la vida. Definir al running como estilo de vida permite pensarlo como una forma estandarizada "de invertir en ciertos aspectos de la vida cotidiana con valor simbólico o social; esto significa que son modos de jugar con la identidad" (Chaney 1996: 44). Y la pluralidad de opciones a las que 
se enfrenta el hombre contemporáneo conduce a adoptar posiciones reflexivas acerca de cada una de sus acciones. Se trata de un mercado que ofrece "tentaciones" (Chaney 1996) cada vez más sofisticadas que en sus continuas renovaciones brindan amplias posibilidades de distinción social. En esa línea, los estilos de vida pueden definirse como "patrones de acción que diferencian a las personas" (Chaney 1996: 16). En consecuencia, se constituyen como instancias claves para la comprensión de la vida cotidiana a partir del establecimiento de fronteras identitarias que cargan de sentido las elecciones personales acerca de lo que somos y lo que hacemos. Los estilos de vida emergen de las prácticas de apropiación, relocalización y resignificación que realizan determinados actores en escenarios particulares. Estos estilos de vida implican además una estilización de la vida cotidiana que conlleva la búsqueda de nuevas sensaciones, de encuentros personales, con los demás y eventualmente con la naturaleza, y en los que juegan un papel relevante las nuevas tecnologías de la comunicación (Sibilia 2008). Además, los actores que se involucran en estos estilos de vida no sólo se ven atravesados por nuevas referencias identitarias sino que administran esas pertenencias de un modo peculiar. En efecto, esas identidades aparecen altamente escenificadas y, con la contundente colaboración de las redes sociales, se muestran como definitorias en el modo de presentación de la persona en la vida cotidiana (Goffman 2001). Un tipo de presentación en la que los actores elaboran detalladas y minuciosas estilizaciones que dan cuenta de pautas éticas y estéticas de gran profundidad.

En este caso, el running en la Argentina puede entenderse como un estilo de vida que es vivenciado y narrado por sus aficionados como un amplio marco de opciones personales. De ese modo, los aficionados controlan sus narrativas acerca del modo en que eligen con completa libertad, por ejemplo, cómo vestirse, alimentarse o relacionarse con los demás. El running puede concebirse entonces como una tecnología de autogobierno (Crossley 2005), dado que los corredores despliegan apuestas reflexivas con la capacidad de adaptarlas a sus proyectos de vida, otorgándoles sentidos particulares. Al tomar a los estilos de vida como algo más profundo que una mera "retórica del mercado del consumidor" (Skkegs 2004: 57) se torna prioritario seguir las formas, usos y trayectorias de esas elecciones. En consecuencia, estos patrones de acción que despliegan densas sensibilidades éticas y estéticas favorecen la articulación de expresiones personales sistemáticas que de ningún modo están disponibles sólo para unos pocos. Sin que pierdan toda relevancia ciertas determinaciones sociales relativas como clase social o género, los estilos de vida favorecerían nociones como individualidad, autoexpresión y autoconciencia. Por supuesto, ese carácter reflexivo dialoga de modo permanente con los imaginarios hegemónicos (estar más delgado y "saludable", la búsqueda de belleza exterior) que también ejercen fuertes presiones en los itinerarios personales, sobre todo en el momento de la elección de un estilo de vida.
El running como estilo de vida contiene un ritual sustancial que se constituye en el eslabón fundamental de las densas cadenas de interacción de los corredores: las carreras. Según Shipway y otros (2013), estas pruebas resultan claves para moldear las normas subculturales y las identidades en el mundo del running. En la misma línea, afirman que "la participación en carreras de larga distancia es una oportunidad para la autorrealización y la autoexpresión, que son beneficios que no siempre se encuentran en la vida cotidiana" (Shipway y otros 2013: 273). Estos sucesivos rituales de interacción (Collins 2005), que pueden implicar además un viaje ${ }^{3}$ previo de miles de kilómetros, producen en los corredores esa energía emocional que le otorga la robustez que caracteriza al universo del running en nuestra contemporaneidad.

Las emociones pueden concebirse como "la energía interna que nos impulsa en un acto, lo que da cierto "carácter» o «colorido» a un acto" (Illouz 2007: 15). Al cargar de energía nuestras acciones, las emociones suponen "al mismo tiempo cognición, afecto, evaluación, motivación y el cuerpo. Lejos de ser presociales o preculturales, las emociones son significados culturales y relaciones sociales fusionados de manera inseparable, y es esa fusión lo que les confiere la capacidad de impartir energía a la acción" (Illouz 2007: 15). En definitiva, "las emociones son aspectos profundamente internalizados e irreflexivos de la acción, pero no porque no conlleven suficiente cultura y sociedad, sino porque tienen demasiado de ambas" (Illouz 2007: 16). La energía emocional que se genera en las carreras opera, en la racionalidad de los corredores, como una poderosa fuerza impulsora para la acción, en este caso entrenar e inscribirse en carreras de forma regular. A través de las redes sociales es en donde los corredores se expresan más elocuentemente acerca de sus sentimientos y emociones. En sus detalladas crónicas y relatos en Facebook, Juan destacó en una oportunidad mientras se preparaba para una ultramaratón en la selva misionera: "Desafío a mí mismo; a mis miedos e inseguridades. Es la búsqueda de la superación misma, es vencer el dolor, la desazón, el desánimo y los imprevistos. Es alimentar esa fuerza interior que te impulsa hacia adelante día a día, es la búsqueda de los sueños". De la eficacia que tengan esas carreras para producir emociones positivas dependerá también la posibilidad de crear y recrear los símbolos de la pertenencia grupal. Incluso en sus rendimientos destacados, muchos corredores optan por destacar la relevancia de pertenecer a un running team. No sólo por los compañeros que "hacen el aguante" alentando a los que corren sino por la fuerza vital que se le asigna al grupo al que pertenecen. Como resaltó Emanuel en una oportunidad, también en Facebook: "Muchas gracias a mis compañeros hoy a pesar de no tenernos mucha fe hicimos una buena carrera a seguir por buen camino y con buena gente se los quiere".

\footnotetext{
3. La importancia de los viajes ha sido destacada por Green \& Jones (2005), quienes los definen como instancias que ofrecen los contextos apropiados para la construcción y autocelebración de identidades y la cristalización de un "ethos" asociado a estas prácticas de ocio.
} 
Una carrera "exitosa" despierta entre los corredores deseos públicos de realizar nuevas apuestas que garanticen una continuidad en este estilo de vida. Las referencias que se obtienen en el campo giran en torno a sentimientos de alegría, orgullo, confianza, fuerza, solidaridad con otros corredores (en particular cuando se pertenece a un grupo de entrenamiento), satisfacción plena. Tanto en las redes sociales como en las conversaciones cotidianas, las impresiones "positivas" luego de una carrera afloran a partir de discursos autocelebratorios de la identidad individual y grupal (los runners en general, los miembros de un running team en particular). Se configura un "contagio emocional" en las todas "situaciones" que hacen fluir palabras, gestos, y ciertas imposturas que dan cuenta de cómo se potencia la solidaridad grupal. Por el contrario, como indica Collins (2005) para los rituales fallidos, una carrera mal organizada, una performance decepcionante sin que medien atenuantes, un circuito poco placentero o cualquier otra fuente potencial de malestar (por ejemplo, incompatibilidad con los compañeros del grupo de entrenamiento), drenan la energía emocional de los corredores. Por supuesto, muchos runners experimentados pueden sufrir decepciones que no consiguen desanimarlos. Como expresó Juan en Facebook, luego de abandonar en un ultramaratón: "una de las experiencias más lindas de mi vida como corredor, el trago amargo, no poder terminarla... el consuelo de tonto: de los 1020 inscriptos habrán finalizado algo así como 150. Lo bueno: habrá que regresar por la revancha". Precisamente, la idea de "revancha", de repetir una carrera frustrante para revertir el sinsabor de un rendimiento decepcionante, impide que se drene la energía emocional en esos casos.

Es en estas interacciones (algunas de ellas cotidianas como los entrenamientos y otras más extraordinarias como las carreras) en donde los corredores experimentan proximidad física y alcanzan un estado de "intersubjetividad intensificada" (Collins 2005: 33), una retroalimentación constante que remite a la noción de efervescencia colectiva de Durkheim. Esas experiencias se pueden objetivar en determinados símbolos, como una remera de la carrera (y que luego se lucirá en los entrenamientos) o la medalla de finisher, que prueba la membrecía a esa comunidad moral de corredores. En definitiva, a partir de estas "situaciones", de esos encuentros temporales y de esa copresencia de cuerpos, los corredores se cargan mutuamente de emociones por el efecto de esas cadenas de encuentros (carreras) pasados y presentes. La energía emocional generada se constituye entonces en un impulso para recrear y hasta incrementar esas cadenas de interacción. Todo ello ocurre en el marco de una atención focal compartida en la que se produce una coordinación recíproca de emociones que tienden a perdurar en torno a una armonía que crea lazos identitarios. Porque los corredores que comparten sus emociones, símbolos, objetos y cierta homogeneidad discursiva, delimitan las fronteras de pertenencia al running, pero también pueden favorecer alteridades internas, entre los corredores "serios" ("verdaderos" corredores) y quienes no lo son.
Según Shipway y otros (2013) estas carreras se constituyen como "un lugar de encuentro e intercambio de experiencias" (Shipway y otros 2013: 272) en donde circula un conjunto de emociones entre las que se destaca "la euforia que se experimenta al final de un evento, que a menudo ilustra el lado positivo del espíritu humano" (Shipway y otros 2013: 272). Es a partir de las carreras en donde los corredores trascienden además la sociabilidad endogámica de sus grupos de entrenamiento, ponen a prueba sus progresos, se fijan objetivos precisos y tienen la posibilidad de compartir su pasión con los no iniciados, por ejemplo los miembros de su familia. Las carreras, en tanto eventos rituales, suelen adquirir una frecuencia periódica y hasta se pueden transformar en sucesos obligados de cada itinerario runner, uno de cuyos rasgos distintivos en el mismo colectivo se construyen a partir de sus elecciones acerca de qué carreras correr. En efecto, la decisión de inscribirse en una carrera y en una determinada distancia le otorga a cada corredor un perfil diferencial, junto con otras variables (como su rendimiento, su estética) que ofrecen una amplia capacidad de apropiación por parte de los aficionados. Y además, la decisión de participar es relevante para "ganar reconocimiento y el respeto de las personas con las que se entrena" (Shipway y otros 2013: 273) durante la semana. En la misma línea, los autores destacan el sentimiento de orgullo que invade a quienes terminan las carreras, que implica un estímulo para reflexionar grupalmente "sobre las experiencias de la carrera y los desafíos enfrentados" (Shipway y otros 2013: 273). Es decir, las carreras no sólo son puntos de referencia obligada para las estrategias de entrenamiento en tanto objetivo deportivo sino que sobre todo, en la concepción nativa de los runners, pueden constituirse (sobre todo los maratones) en experiencias de vida antes que experiencias deportivas, que los cargan de esa imprescindible energía emocional. Todo ello en un contexto general en el que "para muchos aficionados, un elemento importante del running consiste en la ayuda que proporciona para mejorar su propio capital físico y su atractivo, lo que implica una inversión sobre el propio cuerpo" (Shipway y Holloway 2016: 274).

\section{Atletas y runners aficionados}

Shipway y otros (2013) se han referido, desde el contexto británico, a cuatro actores diferenciados en el universo del running, sobre el criterio de mayor o menor compromiso (emocional, temporal, identitario) con esta práctica de alcance global. De ese modo, se refieren en primera instancia a los outsiders deportivos, que son aquellos que sólo participan esporádicamente de algún evento vinculado con el running pero sin un interés específico. Seguidamente, los mismos autores mencionan a los participantes ocasionales, caracterizados por haber adquirido cierto compromiso con su salud y su estado físico y por poseer un conocimiento elemental del mundo del running. En tercer lugar, identifican a los corredores regulares y recreacionales, definidos como aquellos que se relacionan con la práctica de un modo complementario y con otros objetivos deportivos. Finalmente, los autores destacan a los "experimentados 
corredores de larga distancia", distinguidos por pertenecer a una "comunidad" definida con sus reglas y rituales, que habitualmente se traduce además en una membrecía a algún grupo de entrenamiento ("distance running clubs"). Independientemente de las limitaciones de esta clase de tipologías, las características del referente empírico de esta investigación sugieren otros criterios analíticos. En efecto, el colectivo running en la Argentina está compuesto por actores con diverso grado de compromiso con la práctica y adhesión a sus preceptos éticos y estéticos. Además, los corredores suelen transitar itinerarios particulares que pueden llevar, en sus extremos, al abandono del estilo de vida o a un compromiso pleno y radical. Y, como se intenta mostrar en este artículo, el running presenta áreas de contigüidad, ambivalencia y tensión entre diferentes clases de actores y, sobre todo, apropiaciones diferenciales que obedecen a múltiples determinaciones relativas y estilos particulares (Gil 2018).

Más allá de que, en una primera aproximación, podría considerarse al running como una variante de la práctica del atletismo, se trata en realidad de universos diferentes, pero que poseen evidentes y densas zonas de contigüidad. Por supuesto, se sostienen en el mismo ejercicio atlético, pero responden a formas de apropiación y subjetivación diversas por parte de los colectivos igualmente disímiles que los protagonizan. La emergencia del running (en contraste con el atletismo competitivo) no sólo implica un cambio de denominación, un mero snobismo como podría etiquetarse fácilmente. Por el contrario, el running conlleva modificaciones sustanciales en la apropiación de una actividad física puntual -correr- que ha sido llenada de contenidos profundos y diversos. Otro marcado contraste es que las competencias de atletismo de pista no gozan en la Argentina de una adhesión masiva por parte de la población y no logran atraer apoyos económicos significativos por parte del Estado, las empresas o los medios de comunicación. Las contigüidades entre el atletismo y el running están representadas por un conjunto amplio de carreras pero sobre todo por diversos actores "híbridos" (Latour 2007). A través de ellos se expresan, no sin tensiones, conflictos y hasta posturas enfrentadas, los pasajes y diálogos entre ambos universos. De hecho, para muchos actores relevantes del universo running, la distinción con el atletismo parece por demás clara. Uno de los clubes de entrenamiento más notorios y numerosos de Mar del Plata se denomina "de atletismo y running". Incluso su director (como es frecuente en el medio deportivo) llama aficionados a los runners y atletas a quienes se desempeñan en la alta competencia. En término analíticos, los ideales del atleta sacrificado no necesariamente se complementan con los de "una nueva generación de buscadores de aventura para quienes la asunción de riesgos se ha vuelto habitual -y aparentemente incuestionada- en el marco de una serie de experiencias de estilo de vida" (Palmer 2004: 55).

En la Argentina, la organización de competencias atléticas "de calle" parece no tener techo, en gran parte por la gran cantidad creciente de inscriptos. A ello se le agregan los auspicios de importantes empresas, que complementan los recursos que se utilizan para los premios en efectivo destinados a quienes ocupan los primeros lugares de las clasificaciones generales. Ello les permite a no pocos atletas profesionales y semiprofesionales obtener fondos para seguir entrenándose con posibilidades, además de que las performances exitosas pueden atraer sponsors. En el mejor de los casos, los atletas más conocidos con roce internacional son apoyados por multinacionales deportivas y otras empresas de diversos rubros, como suele ocurrir con la suplementación deportiva o la tecnología (por ejemplo la famosa empresa que fabrica los GPS). También algunas compañías locales y regionales, vinculadas o no directamente con el deporte, apoyan a algunos atletas, que lucen esos auspicios en su indumentaria competitiva, que contrasta notoriamente como la remera técnica que entrega la organización de las carreras. Esa indumentaria suele contar inscripciones de los sponsors del evento, junto con la denominación (e inclusive la distancia) de la prueba. La carrera que mejor emblematiza esta contigüidad entre los atletas y los corredores aficionados es el "Maratón, la madre de todas las carreras" (La Nación, 30 de junio de 2016), la prueba paradigmática del atletismo y el running, de 42.195 metros. Más allá de sus componentes míticos que se remontan a la Grecia Clásica, o el dominio sobre la distancia que ejercen los atletas africanos (principalmente de Kenia, aunque también de Etiopía), haber completado (al menos una vez) los 42 kilómetros es un hito relevante, hasta un genuino rito de paso (Van Gennep 2008) en cualquier itinerario runner. Los aficionados suelen considerar que se han transformado en "verdaderos" corredores sólo después de haber corrido un maratón. Además, es habitual que el "santo maratón" (García 2016) se experimente, tal cual lo manifiestan los mismos corredores, como "una experiencia de vida".

Mientras que los aficionados son los que posibilitan ese marco de espectáculo imponente de hasta varias decenas de miles de corredores por las calles de una ciudad, son los atletas de elite los que le otorgan la relevancia en tanto evento deportivo. A tal punto que algunas de esas carreras pueden convertirse en certámenes que otorgan títulos oficiales, como ocurrió en 2018 con el maratón de Buenos Aires, que además se transformó en el Campeonato Sudamericano de la especialidad. Ello permitió que los más destacados corredores del continente, completaran el circuito junto a más de 9 mil aficionados. Poco tiempo antes, el medio maratón de la misma ciudad fue el marco del campeonato argentino de la distancia, en este caso con más de 20 mil corredores en las calles. Pero en líneas generales, algunas de esas carreras constituyen eventos sociales de relevancia y marcas de distinción fundamentales para cualquier aficionado. Inclusive, un corredor poco experimentado hasta puede tener la posibilidad de correr la misma carrera en donde se rompió un record mundial, tal vez demorando el doble del tiempo que el vencedor, que la completa en poco más de dos horas. Pero precisamente este universo de carreras tiene la capacidad de interpelar a los mejores atletas del mundo y a un conjunto cada 
vez más numeroso de corredores globalizados que le da robustez a esa compleja red sociotécnica (Latour 2008) que es el running en nuestra contemporaneidad (Gil 2018). De hecho, el circuito de maratones oficialmente reconocidas por la IAAF (International Association of Athletics Federations) está muy presente para los runners, que en general sueñan con participar en algún major, como se conoce a los seis más importantes del mundo, que se organizan en Nueva York, Chicago, Boston, Berlín, Tokio y Londres. Así es que cada vez que un aficionado participa en un major, es todo un suceso en su grupo de entrenamiento, que difunde la noticia desde sus perfiles oficiales en las redes sociales, y luego informa los detalles de su participación. El más emblemático es el de Boston, ya que se trata del más antiguo de todos los maratones que se corren en la actualidad, dado que se remonta a 1897. Sin embargo, el maratón más famoso y requerido es el de Nueva York.

Por supuesto, un número aún mayor de carreras reafirma constantemente esa contigüidad de ambos universos. Los medio maratones (en la actualidad las competencias de mayor masividad en la Argentina) como las demás carreras de calle (que rondan los 10k) son espacios en donde se refuerza con mayor periodicidad esa contigüidad en las que los atletas comparten el mismo ámbito con aficionados con una amplísima diversidad de ritmos de carrera, estado físico y compromiso con el entrenamiento. Ambos grupos de actores son indispensables para una organización que se monta a partir de esos atletas pero que necesita el marco que le otorgan los aficionados, algunos de importantes rendimientos y otros de un ritmo casi cercano a la caminata. Pero también existe la posibilidad de que aficionados de rendimiento superlativo puedan compartir ritmos de entrenamiento y hasta pasajes de carreras con esos atletas de alta competencia. Por ejemplo, uno de mis informantes centrales tuvo durante mucho tiempo en su foto de perfil en Facebook una imagen de una carrera de $21 \mathrm{~km}$ en la que corría al mismo ritmo que la mejor maratonista argentina del momento, quien además entrena en su mismo running team. Esa capacidad de amplia interpelación que garantiza el running posibilita que incluso los aficionados estén en condiciones de obtener premios y alcanzar cierta notoriedad junto con atletas consagrados, aunque estén lejos de su rendimiento. En efecto, hacer podio forma parte de los horizontes de expectativa de no pocos corredores aficionados. Las carreras suelen establecer un sistema de premiaciones basado en "categorías" por género y edad, en ocasiones con cortes cada cinco años que se extienden mientras haya un número suficiente de corredores inscriptos por cada cohorte eventual. Ello permite que aun estando lejos de los primeros puestos de la clasificación general en una carrera ganada por atletas de elite, es posible acceder a un podio, habitualmente de tres integrantes. Por ejemplo, aquellos corredores que mantienen su constancia en el tiempo (sobre todo luego de los 50 años) pueden empezar a saborear podios por primera vez a medida que avanzan las categorías de edad si es que mantienen o incluso mejoran el rendimiento. La disminución en la cantidad de competidores en esas categorías como también la caída en los ritmos de carrera por cuestiones lógicas de la edad, favorece notoriamente a los más constantes que pueden lograr alguna posición destacada con el transcurso de los años. Otra posibilidad concreta de lograr podios consiste en la estrategia de las parejas o postas mixtas. Algunas competencias, en especial las de trail running, admiten una clasificación por equipos, tanto bajo el sistema de postas como en carrera en equipo, habitualmente en parejas. De hecho, el circuito de carreras que organiza una tienda deportiva de la ciudad de Mar del Plata contempla la modalidad de postas, por categorías, masculina, femenina y mixta. Por lo general, los corredores que no pueden hacer podio en competencias individuales encuentran ese resquicio en las categorías mixtas. Por supuesto esa no es la única motivación, ya que muchas de esas postas o equipos se forman por diverso tipo de afinidad, desde noviazgos hasta amistades, por el solo hecho de compartir esos rituales fundamentales de la sociabilidad runner que son las carreras.

Como se mencionaba más arriba, existen actores híbridos que encarnan esa contigüidad. Formados en el ámbito del atletismo, en ese "deporte de negro", adquirieron su pasión por correr en las pistas atléticas y otras competencias (como el cross-country) ${ }^{4}$, lejos de la notoriedad del running, cultivando ese deporte sacrificado de escasas retribuciones simbólicas y materiales. Algunos de ellos son destacados ex atletas, hoy entrenadores que lideran grupos de entrenamiento más o menos exitosos. En el mejor de los casos entrenan atletas de alta competencia pero su notoriedad y una parte importante de sus ingresos provienen del running, ya que han aprovechado la explosión del fenómeno para organizar exitosos y durables emprendimientos. Ese solapamiento entre el atletismo y el running configura un "combo perfecto", como lo define el líder de un notorio grupo de entrenamiento. Este mismo entrenador y ex atleta de elite destaca "la identificación de los aficionados con los éxitos de los atletas que a veces entrenan con ellos", como ocurre diariamente con los maratonistas de ese equipo. Pero además de ello, a partir de los aficionados del running, dado que muchos de ellos gozan de un elevado capital social, se pueden montar redes de colaboración que redundan en la obtención de sponsors para atletas promisorios, cobertura médica cuando no poseen obra social o apoyos oficiales concretos para distintos emprendimientos. En definitiva, se trata de universos contiguos que tienen una elevada capacidad de retroalimentarse.

\section{Actualidad y crecimiento de las competencia urbanas}

\footnotetext{
4. También denominado oficialmente para atletas federados como campo traviesa, el cross-country se encuentra bajo la órbita de la IAAF, que organiza desde 1973 un mundial de la especialidad con frecuencia bianual y con distancias habituales de 12 kilómetros para los hombres y de 8 kilómetros para las mujeres.
} 
Un tipo de evento que ha tenido un crecimiento exponencial y claramente demostrable es el circuito de competencias urbanas, como ocurre en la capital de la Argentina, donde difícilmente exista un fin de semana sin que se produzca algún evento de estas características. Varias de esas pruebas en la ciudad de Buenos Aires son las de mayor masividad, y además forman parte habitual de la panificación anual de muchos corredores. Los runners marplatenses circulan con una frecuencia constante por esas pruebas, sobre todos los ya mencionados maratón y medio maratón de la ciudad. Las estadísticas, al menos en Occidente, señalan un incremento sostenido desde hace décadas de la cantidad de corredores que participan en las diversas modalidades de carreras, algunas de ellas de una masividad tal que es necesario establecer cupos estrictos, como ocurre el ya mencionado maratón de Nueva York, que se trata además de un evento de un importante impacto turístico. En Brasil, también se registra un crecimiento exponencial del running en la última década. Los inscriptos anuales en carreras de calle llegan casi a los 5 millones y, por ejemplo, en el Estado de São Paulo se organizan anualmente casi 400 carreras (Dias 2017). Para el continente europeo, una publicación colectiva que reúne investigaciones en diversos países (Scheerder y otros 2012) arroja algunos datos ilustrativos de este crecimiento que ya no es novedoso. De hecho, hace más de tres décadas, la incipiente explosión del running en Francia ya había captado la atención académica. Segalen (1994) destacaba que hacia finales de la década de 1980 cerca de 2 millones de franceses corrían con regularidad y participaban de eventos cada vez más masivos y patrocinados por empresas.

Año a año, y no sólo en Buenos Aires sino también en otras ciudades como Mar del Plata y Rosario especialmente, se registra una potente diversificación de carreras "de calle" que han consolidado un calendario repleto de actividades competitivas de las más variadas características, desde las más serias (por la exigencia de la distancias) hasta eventos recreativos, "participativos", de carreras de 3 y 5 kilómetros. En general, los organizadores de las carreras proveen un cuidado kit del corredor, con diversos productos (accesorios, alimentos "saludables") y diseños modernos de indumentaria y en algunos casos con elementos optativos (rompevientos, remeras, vasos térmicos) que hacen variar el costo de inscripción. Las organizaciones más sofisticadas, con mayor capacidad de enrolar sponsors y auspicios oficiales, consiguen montar todo un espectáculo para los corredores, acompañantes y asistentes, con animadores, grupos de música en lugares clave de la carrera, promotoras, stands y, en el mejor de los casos, una cobertura mediática que hasta puede tener repercusión internacional. La logística de la mayor parte de esas carreras (inscripción, dirección técnica) suele estar a cargo de empresas especializadas en eventos de running, que también realizan sus propias competencias en diversos lugares del país, ya sean carreras urbanas como de trail. Mientras que las carreras urbanas suelen tener inscripciones relativamente accesibles (entre 400 y 1000 pesos) ${ }^{5}$, las de trail (sobre todo por su mayor complejidad de organización) suelen demandar costos de inscripción sensiblemente mayores que en un caso alcanza los 800 dólares.

En materia de organización de carreras urbanas, en la ciudad de Buenos Aires es difícil no encontrar alguna competencia "de calle" durante los fines de semana durante el período abril-noviembre. ${ }^{6}$ En la actualidad, estos eventos superan el centenar por año y la cantidad de 500 mil corredores, según las estimaciones oficiales. Con el maratón de la ciudad como exposición ritual cumbre, se organizan también medios maratones y alrededor de un centenar de competencias de distancias menores, entre $5 \mathrm{~km}$ y $15 \mathrm{~km}$, publicitadas con escaso rigor técnico como "maratones". Así es que estas existe un amplio menú de estas "maratones" organizadas por empresas (no sólo deportivas), pero también por diversas entidades, como por ejemplo sindicatos, ${ }^{7}$ asociaciones profesionales, universidades y, sobre todo, entidades de bien público, organizaciones no gubernamentales, fundaciones y hasta una agencia de la Naciones Unidas (ONU) como UNICEF. También existen casos de carreras organizadas por hospitales y entidades confesionales. De ese modo, se llevan a cabo "maratones solidarias" con diversos lemas, tales como correr "por la inclusión", 8 "carrera por la vida" (referida a una campaña por la donación voluntaria de sangre) o "educación para todos", en el marco de acciones para estimular la finalización de la educación secundaria. La concientización sobre la ecología también ha alcanzado al running en el marco de la campaña "La Hora del Planeta" destinada a combatir el cambio climático, impulsada por una ONG global en la que está enrolada la Fundación Vida Silvestre de la Argentina. También se realizan en Buenos Aires carreras con disfraces que apelan a íconos de la cultura popular, como Star Wars, y hasta un evento tematizado por Halloween, sin ningún tipo de impronta competitiva sino más bien artística. Muchas de esas pruebas también admiten la modalidad "kids" y hasta una de ellas, auspiciada por una cadena televisiva infantil, es exclusivamente para niños.

También los clubes son habituales organizadores de carreras, como ocurre con los reconocidos como "cinco grandes" del fútbol argentino. Aunque Independiente de Avellaneda discontinuó la organización de la "Marea Roja",

\footnotetext{
5 . Precios locales que fluctúan de 10 a 25 dólares.

6 . El gobierno de la ciudad le otorga un lugar de relevancia a la promoción y organización de actividades vinculadas con el running. Bajo el slogan "Buenos Aires Corre", en su página web oficial define a esta práctica como "un marco de bienestar y control".

7 . El caso tal vez más emblemático es la "maratón" de 10 kilómetros que organiza el Sindicato Único de Trabajadores de. Edificios de Renta y Horizontal (SUTHERyH) que en 2018 cumplió sus 18 ediciones, remontándose de ese modo a muchos años antes de la explosión del running en la Argentina.

8 . En este caso el Gobierno de la ciudad, a través de Comisión para la Plena Participación e Inclusión de las Personas con Discapacidad, organiza la "Carrera de Todos", con distancias de 5 y $3 \mathrm{k}$.
} 
tanto Boca Juniors, River Plate, Racing de Avellaneda y San Lorenzo de Almagro organizan sus carreras en la actualidad, que en general fluctúan entre los 5 y los $12 \mathrm{~km}$. Lo más habitual es que se trate de eventos que no colocan tanto énfasis en lo competitivo aunque en ocasiones participan atletas destacados. Se trata de eventos de promoción institucional que giran en torno a la identificación con los colores del club, es decir, todo un ritual de reafirmación identitaria y en clara alusión a lo futbolístico. Algunas de esas carreras parten o tienen su llegada en los mismos estadios de fútbol, que se constituyen en una fuente extra de energía emocional para los corredores. Obviamente los circuitos tienen un anclaje barrial que además puede contemplar otras actividades institucionales, como la visita al museo del club. Boca ya realizó siete ediciones de su "maratón" de 12K, ${ }^{9}$ llegando a casi 10 mil inscriptos en 2016 y 2017 . Además de correr por el barrio de La Boca, el evento se promociona a partir de la posibilidad de pisar el suelo de "La Bombonera", su famoso estadio, en uno de sus laterales. Se trata de situaciones ideales para que los corredores se tomen fotos que luego publicarán en las redes sociales. En el caso de River Plate, se propone "enaltecer la marca River de diferentes formas" y en su carrera anual de más de 8 mil inscriptos se transita la pista de atletismo del estadio Monumental. Racing Club, de la localidad de Avellaneda (lindante con la ciudad de Buenos Aires), completó en 2018 su séptima edición de su propia "maratón" de 10 y 5K, también con el atractivo de correr en las puertas de su estadio, El Cilindro. San Lorenzo enmarca la "Maratón Delfo Cabrera"10 (modalidades 10 y 3K) que congrega a casi 2500 corredores en su soñada "vuelta por Boedo", con su política de regreso a su barrio tradicional que implica recuperar los terrenos en donde se hallaba su estadio, El Gasómetro.

Las empresas de indumentaria deportiva (nacionales y multinacionales) también tienen sus propias carreras, aunque habitualmente deleguen la organización en firmas especializadas. Estas competencias cuentan con importantes caudales de corredores y no resulta nada extraño que agoten los cupos de varios miles de participantes. Una compañía nacional asociada a productos e indumentaria "outdoor", por ejemplo, suele completar el cupo máximo de 5 mil corredores para las distancias de 5 y $15 \mathrm{k}$ en la zona de Palermo, a la que acceden mediante el pago de una inscripción accesible y un completo kit del corredor. De modo similar, en el marco de su inserción comercial en la Argentina, una empresa de origen norteamericana especializada en running se sumó a la organización de carreras desde 2017, también en la zona de Palermo. Sostenido en su concepto de "run happy", esta carrera que alberga 2 mil corredores (los cupos se agotan) postula la opción por correr como una experiencia divertida y compartida con amigos. De allí que se coloque especial

\footnotetext{
9. La distancia de 12 kilómetros es un homenaje al "jugador №12, como se conoce a su hinchada de fútbol.

10. Célebre atleta argentino que en 1948 obtuvo la medalla de oro en maratón en los Juegos Olímpicos de Londres.
}

énfasis en la música en vivo durante el recorrido (violinistas, batucadas, DJs). Esta empresa afincada en Seattle desde 1914 proclama su compromiso con el medio ambiente, ya que utiliza materiales biodegradables y su fábrica utiliza energías y recursos renovables.

Más allá de todas estas carreras, son los maratones y los medio maratones los eventos que expresan en toda su dimensión la robustez del running. Tanto por la masividad que suelen concitar estas carreras como la sofisticación de la logística de organización (circuito, promoción, kit del corredor, hidratación durante la carrera) se constituyen en los rituales de interacción por excelencia para cualquier corredor. Es generalmente a partir del medio maratón cuando la opción por correr como un estilo de vida se transforma en una experiencia vital distintiva capaz de generar esa energía emocional necesaria para proyectar incluso objetivos más ambiciosos, como podría ser el maratón o hasta un ultramaratón (cualquier distancia que supere los 42.195 metros). El medio maratón de la ciudad, que se suele organizar en agosto o septiembre de cada año, es promocionado como la competencia atlética de mayor masividad de América Latina. Desde 2014 supera holgadamente los 20 mil inscriptos, frente a los 17.700 de 2013, los 14.589 de 2012 y los 13.500 de 2011. Sin embargo, recién hacia 2005 este medio maratón comenzó a mostrar cifras de inscriptos superiores a los 4 mil corredores y todavía no había incorporado los principales rasgos de la estética y logística del running. Sin demasiada promoción y escasa presencia e inversión de empresas deportivas, ni siquiera se entregaba la "remera técnica" que comenzaba a ser habitual en este tipo de carreras. Muchos menos existía el kit del corredor.

El Maratón de Buenos Aires, que suele realizarse entre los meses de septiembre y octubre, se ha estabilizado en los últimos años en una cifra de alrededor de 10 mil corredores, mientras que hacia 2011 hubo un total de 7200 inscriptos. Cuando en 1984 se organizó la primera edición sólo 18 atletas participaron, aunque en 1985 ya fueron 149. Ambas competencias, las dos más importantes "de calle" del país, están experimentando un amesetamiento en el número de corredores, por lo que una lectura simplista podría sugerir un agotamiento en el crecimiento exponencial del fenómeno. Sin embargo, ello ni siquiera puede aplicarse al ámbito de las carreras urbanas, ya que por otro lado ha crecido un circuito complementario en la Ciudad de Buenos Aires que permite que se corran hasta tres medio maratones con regularidad, además de otro más en la vecina localidad de Vicente López, todos ellos de importante convocatoria. Una de esas carreras es organizada por una empresa multinacional de indumentaria de origen japonesa en la que participan 4 mil corredores y las dos restantes por una empresa especializada en eventos de running cuyo lema es "sólo pensá en correr". Esos eventos son patrocinados en la actualidad por otras dos compañías multinacionales de indumentaria, una norteamericana y otra japonesa (ese evento contó con 8 mil inscriptos), ambas de gran renombre en calzado deportivo. 
A todo este panorama debe sumársele hasta una carrera de la distancia poco convencional de 30 kilómetros que admite distancias "menores" de $21 \mathrm{~K}$ y $10 \mathrm{~K}$. Esta última competencia se organiza en el sur de la ciudad, un área poco utilizada para este tipo de eventos, que se suelen concentrar en la zona norte y sobre todo en los bosques de Palermo y los Rosedales y Puerto Madero, que además son los lugares en donde se observa una mayor afluencia de corredores y grupos de entrenamiento. Los 30K fueron pensados para aquellos corredores que empiezan a sentir que un medio maratón ya no constituye un esfuerzo importante pero que todavía no están en condiciones de planificar un maratón. Esta carrera ha experimentado una adhesión importante, aunque lejos de las cifras de otras competencias. Además, se suele correr en el mes de junio con temperaturas sensiblemente bajas, aun para los estándares estoicos de los corredores acostumbrados a entrenar en condiciones de mayor rigor. Esta innovación presenta la cualidad de posicionarse en la distancia crítica de los 30 kilómetros, que habitualmente se denomina "el muro", por tratarse de un momento en el que el corredor siente de manera repentina el esfuerzo del trayecto realizado cuando todavía falta una distancia considerable por recorrer. Se trata de una barrera física y psicológica entre los 30 y 35 kilómetros que remite a un descenso brusco en la energía del corredor. Las explicaciones médicas señalan que ello se produce cuando el cuerpo modifica el sistema mediante el cual obtiene energía. En determinado momento se deja de utilizar eficientemente las reservas de glucógeno que se almacenan en el hígado y en los músculos, razón por la cual se aconseja consumir un volumen considerable de hidratos de carbono los días previos a una carrera para extender al máximo posible la aparición del "muro". Por supuesto, eso dependerá de cada corredor, su entrenamiento, el ritmo de carrera, la hidratación, el consumo de hidratos de carbono durante la carrera (frutas, gomas y geles energéticos) y el peso del corredor, entre otras variables.

Mar del Plata, el lugar de estudio predominante de esta investigación, también es otro ejemplo que acompaña en la misma sintonía de lo que ocurre en la capital del país. A la estabilización de una serie de carreras con cierta tradición, se le están sumando paulatinamente otras competencias de gran masividad y que además logran apoyos importantes de los auspiciantes. Sindicatos, clubes y empresas se han incorporado con cierta constancia en la organización de carreras en diversos sectores de la ciudad, aunque casi siempre sobre su extensa franja costera. También en localidades vecinas como Miramar, Chapadmalal y Nicanor Otamendi se realizan distintos certámenes atléticos, aunque muchos de ellos en la modalidad cross-country y bajo los parámetros organizativos y estéticos de las competencias atléticas anteriores a la explosión del running. En varias de estas estas carreras se entrega un kit de corredor modesto, no se cuenta con auspicios de relieve y las inscripciones son mucho menos onerosas. Algunos de esos eventos (como una carrera que se relizaba en las canteras de la vecina localidad de Batán o una nocturna en la zona de Laguna de los Padres) se dejaron de organizar pero siempre aparecen nuevos intentos de estabilizar competencias. En efecto, el nutrido calendario de carreras de Mar del Plata y su zona de influencia no goza de la sofisticada logística de la mayor parte de las competencias celebradas en Buenos Aires. Y tampoco todas las que se organizan han logrado una continuidad en el tiempo como para transformarse en tradiciones para los runners locales y potenciales visitantes. Sólo algunos pocos eventos, como el Maratón de la ciudad, consiguen generar el clima característicos de los más importantes rituales de interacción del running. También una tienda deportiva local organiza un circuito de carreras de $15 \mathrm{~km}$ por etapas combinando los rasgos geográficos de la ciudad. La mayor parte de esas pruebas, ampliamente difundidas en las redes sociales y por correo electrónico a la comunidad runner, concitan una marcada y creciente masividad. Para 2019 esta ciudad contará con tres medio maratones, uno auspiciado por el gobierno provincial ("Buenos Aires corre"), otro organizado por la mencionada cadena local de tiendas deportivas y el restante en el marco del maratón de la ciudad.

En efecto, el evento de mayor relevancia que consagra la contigüidad entre el atlestismo y el running es el "Maratón Ciudad de Mar del Plata" que ya lleva 29 ediciones. Ese maratón era organizado históricamente por la Federación Marplatense de Atletismo pero fue interrumpido en la primera década de este siglo hasta que se retomó su organización en 2012. A partir de allí, el Ente Municipal de Deportes (EMDER) la encuadró dentro de los nuevos parámetros del running, por ejemplo proporcionando los kits del corredor para los inscriptos o instaurando premiaciones por categorías de edad. En esa sintonía, la promoción del evento estuvo enmarcada, como lo seguiría siendo con más énfasis en los años posteriores, como un típico acontecimiento del running. Para esa nueva edición de 2012 participaron aldededor de 2400 corredores en las tres distancias de $10 \mathrm{~K}, 21 \mathrm{~K}$ y $42 \mathrm{~K}$. El aumento de inscriptos en las sucesivas ediciones ha sido significativo. En la edición de 2013 participaron alrededor de 3500 corredores, mientras que en 2014 lo hicieron algo más de 7 mil y en 2015 alcanzó los 8 mil participantes. La versión de 2016 registró una marcada merma de corredores (rondaron los 6 mil), debido a los cambios organizativos que llevaron a que se confirmara la fecha de realización de la carrera con escasa antelación. A partir de ese año, la municipalidad (bajo otra administración política) tercerizó en privados mediante una licitación la organización de la carrera. En 2017 más de 9 mil corredores participaron en las tres distancias y en 2018 se agotó el cupo de 9 mil corredores con más de una semana de anticipación.

\section{Las carreras, los medios y las redes sociales}

Los medios masivos de comunicación y en especial las redes sociales cumplen un papel fundamental en cristalizar la relevancia de estas competencias. Por un lado, el running 
cuenta con amplios canales de difusión, desde revistas especializadas, blogs, programas televisivos en cadenas nacionales e internacionales y programas de radio. Redes sociales como Facebook son fundamentales en la actualización informativa, difusión de fechas e instalación de carreras nuevas. Esta red permite además promocionar las virtudes geográficas de los recorridos que es otro de los ingredientes fundamentales para construir una "buena carrera". En efecto, una competencia debe estar bien fabricada, adquirir la robustez necesaria para lograr la durabilidad de todos sus componentes, tanto humanos como no humanos (Latour, 2008). La posibilidad de enrolar corredores (los amateurs y los de elite) sponsors importantes (en la provisión de indumentaria, bebidas, alimentación, entre otras posibilidades) y burocracias locales (la obtención de permisos para montar los circuitos, la declaración de interés, sostén en la difusión y apoyo logístico) resultan claves para esos objetivos. Herrick (2015) también ha destacado que aquello que denomina como eventos de running de participación masiva ("mass participation running events"), requieren del apoyo conjunto de "actores, conceptos, ideas, estrategias políticas y planificación gubernamental urbana" (Herrick 2015: 310). Para ello montan un densa red compuesta por "atletas de elite, la masa de participantes, funcionarios municipales, figuras políticas, comentaristas de deportes de televisión, periodistas, diplomáticos, empresas patrocinadoras, agencias de turismo, compañías de gestión deportiva y buscadores de talentos, organizaciones benéficas y organizaciones de desarrollo y ONGs" (Herrick 2015: 310). En la misma dirección, se destaca que se trata de un "sistema profundamente emprendedor" (Herrick 2015: 310) que apunta a la gestión privada y a la participación masiva de los eventos deportivos en las ciudades. Precisamente, la realización de este tipo de acontecimientos deportivos interpela las lógicas de gestión de los municipios, que suelen enfrentar no pocos dilemas en su autorización y/o promoción. Por ejemplo, resulta frecuente, y en ese sentido las redes sociales son un canal de expresión privilegiado, que los cortes ocasionados en las ciudades para la organización de estos eventos despierte cuestionamientos severos. En ciudades como Buenos Aires, en donde los cortes de calle a cargo de movimientos sociales, sindicatos y partidos políticos operan con frecuencia semanal, las interrupciones al tránsito en esas mañanas de domingo reciben quejas de los automovilistas y otros sujetos que se refieren a estos eventos como "piquetes VIP"11 o "piquetes oficiales".

Definitivamente, para lograr el éxito de una carrera, los organizadores deben provocar constantes y amplios "interesamientos" (Callon y Law 1998) para construir una red sólida con capacidad de mantenerse en el tiempo. En ese sentido, algunas carreras (en especial las del circuito de trail running) desarrollan un marketing orientado a establecer relaciones cercanas con los consumidores

\footnotetext{
11. El piquete es una forma habitual de protesta social que se realiza en la Argentina desde la década de 1990 y que consiste en el corte de calles y rutas.
}

(corredores). Ello configura un conjunto de innovaciones que, sobre todo en los eventos de alto perfil, caracterizan el marketing deportivo en el contexto de la globalización y las redes sociales (Thompson y otros 2016). La participación en las carreras suele adquirir un denso correlato virtual por el modo en que los corredores espectacularizan esa participación en las redes, sobre todo si además se obtuvo un resultado descollante (un buen tiempo, un podio). Ariel, un aficionado habituado a esos podios, actualiza sus resultados y tiempos en las redes sociales de modo permanente, y de un modo similar a otros corredores, con frases tales como "quedando 3ro en categoría 25-29años y 18gral, contento con el resultado, seguimos". Esas valoraciones del propio rendimiento van acompañadas por fotos de la carrera en las que aparecen ellos mismos, antes, durante y después de la competencia. No resulta nada extraño que los runners escriban detalladas crónicas de su participación, proporcionando no sólo datos puntuales (tiempos, posición general, posición en categoría por edad) sino relatos introspectivos que pueden combinar confesiones, postulados filosóficos o reflexiones generales sobre la vida a partir de la experiencia distintiva de correr. Y cuando se producen éxitos deportivos para destacar en un grupo de entrenamiento, se recibe un gran número de felicitaciones de propios y ajenos, pero sobre todo de sus integrantes, con intervenciones en las redes sociales tales como: "qué orgullo ser parte de este equipo!".

Muchos corredores también acostumbran subir videos a sus perfiles sobre los preparativos de las competencias, las instancias previas y hasta del desarrollo de la carrera. En definitiva, el running se configura como una "bella red sociotécnica" (Latour 2008) de la que forman parte actores humanos y muchos otros no humanos, tales como las tecnologías de la comunicación, las empresas multinacionales, los medios de comunicación y el medio ambiente, entre muchos otros componentes de esa red. De este modo, los organizadores de las pruebas estimulan la creación de vínculos con los corredores y entre los corredores, favoreciendo un clima de diálogo y participación continua. Esa interacción facilita la generación de conexiones emocionales con las carreras. Ello ocurre frecuentemente cuando, por ejemplo, se publican las fotos de los corredores, que pueden sentirse protagonistas de ese evento e incorporarlo como hito significativo en su biografía runner. Luego de cada evento, los participantes dejan sus opiniones sobre los diversos aspectos organizativos, que pueden ser contestados desde la organización. Un uso productivo de estas herramientas permite acceder a las demandas habituales de los participantes (por ejemplo hidratación, entrega de elementos promocionales) y estar alerta a los diversos aspectos de la organización. Así, en el mejor de los casos, se logra cultivar un sentimiento de pertenencia a lugares, momentos y hasta determinadas empresas, cuando una carrera adopta el nombre de quien la patrocina (Eagleman 2013).

A través de esta clase de estrategias, algunos organizadores 
apelan a los influencers, quienes promocionan las virtudes del estilo de vida runner en general y de una carrera en particular. Estos influencers, en tanto promotores de ciertos estilos de vida (Abidin 2017), operan como "intermediarios de estas «sensaciones personales» cuando incorporan productos para la venta en sus vidas personales y representan estilos de vida a través de la publicidad. Su enfoque altamente personalizado torna ambigua la distinción entre sus vidas privadas y públicas, de modo que pueden aprovechar la conexión que han establecido con sus seguidores para provocar un impacto en las decisiones de compra" (Abidin 2017: 166). Se trata de sujetos que tienen la capacidad -o se cree que tienen- de influenciar a los potenciales consumidores, en este caso para la elección de determinadas carreras, cierta indumentaria o incluso hábitos alimenticios. ${ }^{12}$ Aunque esa práctica casi ha desaparecido en las promociones de las carreras en las redes sociales, los organizadores han recurrido en ocasiones a ciertas celebridades (generalmente del mundo de la farándula) como difusores de sus competencias. Muchos son sujetos que se volcaron al running y promocionan su nueva afición, siendo enrolados por los organizadores y eventualmente empresas de indumentaria como caras visibles de sus campañas.

Para crear ese clima de carrera importante, las redes sociales son una herramienta central. Una efectiva utilización de estos nuevos medios genera crecientes horizontes de expectativa a medida que se acerca la fecha de una carrera, sobre todo de aquellos organizadores que tienen capacidad de generar noticias durante todo el año. Por eso es que el uso de plataformas de comunicación efectivas favorece el desarrollo de un marketing relacional, con el objetivo central de "retener clientes al lograr la satisfacción mutua a largo plazo entre una organización y sus clientes" (Abeza y otros 2013: 120). En ocasiones durante todo el año se monta una compleja trama de presencia digital. Así se va construyendo el clima previo que ayuda a cristalizar a esa carrera como una tradición irrenunciable en el currículum de cualquier corredor. También, a medida que se acerca la fecha de realización de la carrera, se van confirmando las presencias de las celebridades del running y las novedades organizativas, como el kit del corredor, que será el souvenir de la experiencia por vivir y eventualmente indumentaria de futuros entrenamientos. De esta manera, la utilización de las diferentes plataformas virtuales expande esos rituales de interacción, tanto en el plano espacial como el temporal. Ello se hace aún más evidente cuando en algunos casos las carreras pueden ofrecer un streaming en vivo, accesible a familiares y amigos de los corredores, pero también a futuros participantes. En el mejor de los casos, algunas de esas pruebas son difundidas en programas especializados, como ocurre con algunas pocas seleccionadas en la emisión

12. Como describe Hijós (2017), los "fitfluencers" cumplen un papel fundamental en marcar, en este caso a través de los medios digitales, el camino hacia una "vida sana", sostenida en el ejercicio y la alimentación, en tanto "fenómeno global en expansión que atrae a miles de seguidores, y transforma a sus protagonistas en celebridades y objetos de culto" (Hijós 2017: 7). internacional ESPN Run. Aunque la gran mayoría, al menos, consigue que diversas páginas y blogs especializados en running se hagan eco de la competencia. En definitiva, las redes sociales constituyen herramientas imprescindibles para estos deportes con nichos de mercado tan delimitados y que gozan de una presencia tan marginal en los medios masivos de comunicación más tradicionales. Sin embargo, son disciplinas que manejan clientelas fieles e intensas, con gran capacidad de consumo y de presencia periódica en un considerable número de eventos.

\section{A modo de conclusión}

A lo largo de este artículo se ha intentado describir detalladamente el lugar que ocupan las carreras en el marco de la sociabilidad runner. Pero sobre todo, se ha mostrado la relevancia que tienen en tanto ritual de interacción fundamental para darle forma a un fenómeno global todavía en plena expansión. En este texto sólo se ha presentado una parte del fenómeno en la Argentina, ya que no se han considerado las carreras de trail. Este género "de aventura" reproduce la mayor parte de las lógicas de las carreras urbanas pero presenta otras aristas salientes y diferenciales (turísticas, ecológicas) que trascienden la diferencia evidente entre correr por las calles de una ciudad o por una montaña cordillerana. Las carreras, además de ser poderosas fuentes de energía emocional para los corredores, se constituyen en el espacio clave de expresión de la estética y moralidad runner. La exposición de los corredores en esas plataformas digitales, en las que narran detalladamente su preparación para cada competencia y sus experiencias en esos eventos, resumen algunos de los aspectos más notorios del modo en que se experimenta su identidad. La extensa variedad de carreras evidencia la amplísima capacidad de interpelación de este estilo de vida en el que cada corredor puede manejarse entre un amplio menú de carreras, desde una más "glamorosa" (y por ende más cara su inscripción y los gastos eventuales de traslado) patrocinada por una empresa multinacional hasta una más modesta organizada por un club barrial o una asociación atlética de una pequeña ciudad. Las carreras, además, están concebidas para que corredores con grandes diferencias de rendimiento transiten los mismos circuitos, desde distancias de 5 kilómetros hasta los extenuantes maratones y ultramaratones. Allí es donde se advierten los rasgos distintivos del running en contraste con el atletismo de alta competencia, pero también sus áreas de contigüidad y continua retroalimentación.

Por todo lo expuesto, las carreras son una excelente puerta de entrada para comprender la robustez con que está construido el universo running. A partir de estos eventos que son vivenciados por los corredores como experiencias de vida, la opción virtuosa por correr cobra plena dimensión. Y por supuesto, las carreras favorecen una marcada estilización de la experiencia atlética y un potente fuente de energía emocional para los aficionados. Además, estos eventos permiten apreciar, al menos 
parcialmente, que el running es una poderosa tecnología de autogobierno (Crossley 2005), dado que los corredores ejercitan sus apuestas reflexivas, cargándolas de sentido para cada proyecto personal. La lógica de organización y participación en las carreras muestra que, como ya lo había advertido Segalen (1994), todos pueden sentirse como corredores que están haciendo algo importante con su vida, reafirmando en cada experiencia una opción bella y virtuosa. Es decir, las carreras permiten apreciar una de las características salientes que explican una expansión del running que no parece conocer límite. Finalmente, las carreras también nos enfrentan a desafíos urbanísticos y de políticas públicas (Herrick 2015). En efecto, estos eventos se pueden transformar en marcas identitarias de un espacio urbano, en una atracción turística o en muestra de la capacidad de gestión pública. Las ciudades pueden encontrar en la promoción de estas actividades, y de otras similares, oportunidades para posicionarse como espacios urbanos modernos y que estimulan estilos de vida "saludables", además de cristalizar tradiciones deportivas que les permitan reclamar un signo de distinción.

Mar del Plata, 28 de marzo de 2019

\section{Bibliografía}

Abeza, G., N. O'Reilly, I. Reid (2013). Relationship marketing and social media in sport. International Journal of Sport Communication, 6: 120-142.

Abidin, C. (2017). Influencer extravaganza. Commercial "Lifestyle" Microcelebrities in Singapore. En L. Hjorth, H. Horst, A. Galloway y G. Bell (eds). The Routledge Companion to Digital Ethnography. New York and London: Routledge, 158-168,

Bennet, A. (1999). "Subcultures or neo-tribes? Rehinking the relationship between youth, style and musical taste". Sociology, 33 (3): 599-617.

Callon, M., J. Law (1998). De los intereses y su transformación. Enrolamiento y contraenrolamiento. En J. Domenech y F. Tirado (eds). Sociología simétrica. Ensayos sobre ciencia, tecnología y sociedad. Barcelona: Gedisa, 51-61.

Collins, R. (2005). Interaction Ritual Chains. Princeton University Press: Princeton y Oxford.

Crossley, N. (2005). "Mapping Reflexive Body Techniques: On Body Modification and maintenance". Body \& Society, 11(1): 1-35.

Chaney, D. (1996). Lifestyles. London: Routledge.

Dias, C. (2017). "Corrida de rua no país do futebol". Recorde, 10 (1): 1-32.

Eagleman, A. (2013). Acceptance, motivations, and usage of social media as a marketing communications tool amongst employees of sport national governing bodies. Sport Management Review 16: 488-497.

García, S. (2016). Correr para vivir, vivir para correr. Buenos Aires: Debate.
Giddens, A. (1995). Modernidad e identidad del yo. El yo y la sociedad en la época contemporánea. Península: Barcelona.

Gil, G. (2015). "Cuerpos que corren y viajan. El trail running en la Argentina". En Jornadas de Sociología de la UBA, 13 al 17 de julio.

Gil, G. (2018). "Deporte y estilos de vida. El running en Argentina". Antípoda. Revista de Antropología y Arqueología, 30: 43-63.

Goffman, E. (2001). La presentación de la persona en la vida cotidiana, Amorrortu, Buenos Aires.

Green, C. y Jones, I. (2005). "Serious leisure, social identity and sport tourism". Sport in Society, 8(2): 198-217.

Herrick, C. (2015). "Comparative urban research and mass participation running events: methodological reflections". Qualitative Research, 15(3): 296-313.

Hijós, M. (2017). "«Fitters», «paleo» y «veggies»: nuevas formas de clasificar lo comestible. Educación Física y Ciencia, 19(2), e034. https://doi.org/10.24215/23142561e034.

Illouz, E. (2007). Intimidades congeladas. Las emociones en el capitalismo. Buenos Aires: Katz.

Latour, B. (2007). Nunca fuimos modernos. Ensayo de antropología simétrica. Buenos Aires: Siglo XXI.

Latour, B. (2008). Reensamblar lo social. Una introducción a la teoría del actor-red. Buenos Aires: Buenos Aires.

Marcus, G. (1995). Ethnography in/of the World System: The Emergence of Multi-Sited Ethnography. Annual Review of Anthropology, 24: 95-117.

Palmer, C. (2004). Death, danger and the selling of risk in adventure sports. En B. Wheaton (ed). Understanding Lifestyle Sports. Consumption, identity and difference. Londres: Routledge.

Scheerder, J., K. Breedveld y J. Borgers (eds.) (2015). Running across Europe. The Rise and Size of One of the Largest Sport Markets. Hampshire: Palgrave Macmillan.

Segalen, M. (1994). Les enfants d'Achille et de Nike. Une ethnologie de la course à pied ordinaire. Paris: Éditions Métailié.

Shipway, R., I. Holloway (2016). "Health and the running body: Notes from an ethnography". International Review for the Sociology of Sport, 51(1): 78-96.

Shipway R., I. Holloway, I. Jones (2013). Organisations, practices, actors, and events: Exploring inside the distance running social world. International Review for the Sociology of Sport, 48(3): 259-276. DOI: 10.1177/1012690212442135.

Sibilia, P. (2008). La intimidad como espectáculo. Buenos Aires: Fondo de Cultura Económica.

Skeggs, B. (2004). Class, Self, Culture. London: Sage.

Thompson, A., M. Andrew, S. Gee, A. Geurin (2016) Fans' Perceptions of Professional Tennis Events' Social Media Presence: Interaction, Insight, and Brand Anthropomorphism. Communication \& Sport, 5 (5): 579-603.

Van Gennep, Arnold (2008) Los ritos de paso. Madrid: Alianza. 